Saudi Journal of Oral and Dental Research

Abbreviated Key Title: Saudi J Oral Dent Res

ISSN 2518-1300 (Print) |ISSN 2518-1297 (Online)

Scholars Middle East Publishers, Dubai, United Arab Emirates

Journal homepage: http://scholarsmepub.com/sjodr/

\title{
Characteristics and Distribution of Facial Pain Related to Dental Conditions
}

Noor Ahmed Alabdullah $^{1 *}$, Ayat Salman Mohammed Alabdullah ${ }^{2}$, Maha Hassan Al Somali ${ }^{3}$, Mrwah Jwad Ali Alojan ${ }^{4}$ Mawaddah Mousa Almousa ${ }^{5}$, Ghadeer Abdulqader Saati ${ }^{6}$, Osama Ahmad Almassri ${ }^{7}$, Fatima Sultana ${ }^{8}$

${ }^{1}$ Luluah Dental Clinic, Jeddah, KSA

${ }^{2}$ Family Dental Centre, AlAhsa, KSA

${ }^{3}$ Juman Medical Centre, Al Khobar, KSA

${ }^{4}$ Beauty Zone Poly Clinic, KSA

${ }^{5}$ Amani Albsmah Clinic, Makkah, KSA

${ }^{6}$ Dr. Qari Specialist Dental Clinic, Jeddah, KSA

${ }^{7}$ The clinics Medical center, Riyadh, KSA

${ }^{8}$ Research Centre, Riyadh, KSA

DOI: $10.36348 /$ SJODR.2019.v04i12.002 $\quad$ | Received: 06.11.2019| Accepted: 19.11.2019| Published: 09.12.2019

*Corresponding author: Noor Ahmed Alabdullah

\section{Abstract}

The head face, temporomandibular joint, and the maxillary and the mandibular muscles consist of the trigeminal system and hence the pain is more prevalent in these structures. The orofacial pain affects the patient's quality of life dramatically. In order to manage the orofacial pain disorders, the clinician need to be familiar with different types of etiologies and characteristics of facial region. In order to attain safe and best therapeutic results, the trigeminal system plays a significant role. It is a cross-sectional descriptive study aimed at evaluation of prevalence and characteristics of dental orofacial pain. The Diagnostic Criteria of Temporomandibular Disorders (DC/TMD) questionnaire was disseminated online, limiting participants to those who resided in Saudi Arabia. A total of 226 participants completed the survey. Axis I related disorders were diagnosed in 195 patients, whereas Axis II related disorders were diagnosed in 36 of patients. This study showed high prevalence rate and an immense range in pain intensity with commensurate and often significant impact on the quality of life. The Research Diagnostic Criteria for Temporomandibular Disorders (RDC/ TMD), has been widely used globally to estimate prevalences of TMD. Additional research is necessary to establish comprehensive classification schemes for all patients with orofacial pain.

Keywords: Temporomandibular joint, orofacial pain disorders, trigeminal system, TMD.

Copyright @ 2019: This is an open-access article distributed under the terms of the Creative Commons Attribution license which permits unrestricted use, distribution, and reproduction in any medium for non-commercial use (NonCommercial, or CC-BY-NC) provided the original author and source are credited.

\section{INTRODUCTION}

The orofacial pain disorders deal with the management of pain by prevention, diagnosis, treatment, and rehabilitation. This kind of disorder is associated with the pain that arises due to varied reasons like pain after surgery, pain due to malignancy, and syndromes like temporomandibular joint disorder pain, headache, or neuropathic pains. The orofacial pain includes the pain associated with the face, neck, and head.

Orofacial pain has numerous origins that include muscle, joint, sinus, pulpal, periodontal, and vascular sources [1]. Orofacial pain gravely impacts the patients' quality of life [2]; Pain may be relapsing and remitting, constant, or aggravated by certain factors [3]. Additionally, it is reported to be among the top reasons to visit a healthcare provider [4]. The oral health care providers, including the dentists, undoubtedly treat patients with orofacial pain frequently.

Among the most common etiologies for orofacial pain are temporomandibular disorders (TMDs). TMDs have a significant impact on activities of daily living [5]. Many issues concerning the etiology, diagnosis, and treatment of temporomandibular disorders (TMD) are still unclear. A thorough study of TMD is required by using standardized classification systems. The Research Diagnostic Criteria for Temporomandibular Disorders (RDC/TMD) and the updated version, now called Diagnostic Criteria for Temporomandibular Disorders (DC/TMD), are the current references for appraisal of TMDs [6]. However, not all patients with orofacial pain have TMD. It is thus crucial for health practitioners to determine the 
abortifacient factors behind orofacial pain in order to provide the essential treatment, enhance the quality of life, and reduce the social and economic impacts of this state.

To the best of our knowledge, there are no estimates on the presence and severity of orofacial pain attributed to dental disorders in Saudi Arabia. In this study, we aim to evaluate the prevalence and nature of orofacial pain among residents of Saudi Arabia, relying on a locally-adapted, culturally-relevant version of the DC/TMD questionnaire.

\section{METHODOLOGY}

This was a cross-sectional descriptive study aimed at evaluating the prevalence and characteristics of dental orofacial pain in Saudi Arabia. Electronicallydisseminated case report forms were employed.

The Diagnostic Criteria of Temporomandibular Disorders (DC/TMD) questionnaire was first formulated in the English language. This is then modified, translated to Arabic, validated, and tested in a group of bilingual volunteers who provided feedback on the interpretation of each question [6]. The questionnaire was disseminated online, limiting participants to those who resided in Saudi Arabia.

The questionnaire is bifurcated into two parts: Axis I is based on guidelines for oral history taking and clinical assessment, while Axis II deals with the evaluation of pain-related impairment and other psychological features, such as depression and somatization levels.

According to the Saudi Arabian General Authority for Statistics, the overall population size of residents of Saudi Arabia is about 33 millions. For this study, we assumed a prevalence of $5 \%$ and a precision of $3 \%$ for the $95 \%$ confidence interval. This gave us a required sample size of 203. The questionnaire was disseminated on several social media platforms. The questionnaire message was re-distributed at regular intervals, being posted at least once a day from select Saudi Arabian influencer accounts. Optimum post time was determined using each social media platform analytics tools. The questionnaire was withdrawn when more than 203 complete responses were received, and participants were interviewed. The study was conducted according to the Declaration of Helsinki, and our institutional review board approved the protocol.

Participants received none or one or more of the following groups of axis I diagnoses on one or both sides: muscle disorders (group I, with possible subgroups: IA: myofascial pain; and IB: myofascial pain with limited opening), disk displacement (group II, with likely subgroups: IIA: disk displacement with reduction; IIB: disk displacement without modification with limited mouth opening; and IIC: disk displacement without mitigation, without limited opening), or arthralgia, osteoarthritis, and osteoarthrosis (group III, with possible subgroups: IIIA: arthralgia-pain and tenderness in the joint capsule and/or the synovial lining of the TMJ; IIIB: osteoarthritis_pain and tenderness in the joint capsule and/or the synovial lining of the TMJ and coarse crepitus in the TMJ; and IIIC osteoarthrosis-coarse crepitus in the TMJ and/or tomograms showing pathology in the TMJ. Following an algorithm included in the instructions for RDC/TMD, it was possible to classify the participants into the following groups on axis II: involved painrelated impairment (Graded Chronic Pain Severity, from 0: no TMD pain in the prior 6 months, to IV: high disability - severely limiting), depression, and somatization (levels: standard, moderate, severe).

Continuous variables that followed Normal distribution were summarized as means with standard deviation (SD). Categorical variables were summarized as frequencies. Box-Cox plots and histograms were used to screen-out outliers. Fisher's exact test with a two-tailed p-value $<0.05$ was used to indicate significance.

\section{RESULTS}

A total of 226 participants completed the survey. Median age (interquartile range) and female: male distribution was 35.0 (28-48) years and 2.7:1, respectively. Overall, $73 \%$ of participants rated their health as very good or excellent, and $66 \%$ rated their oral health as very good or excellent.

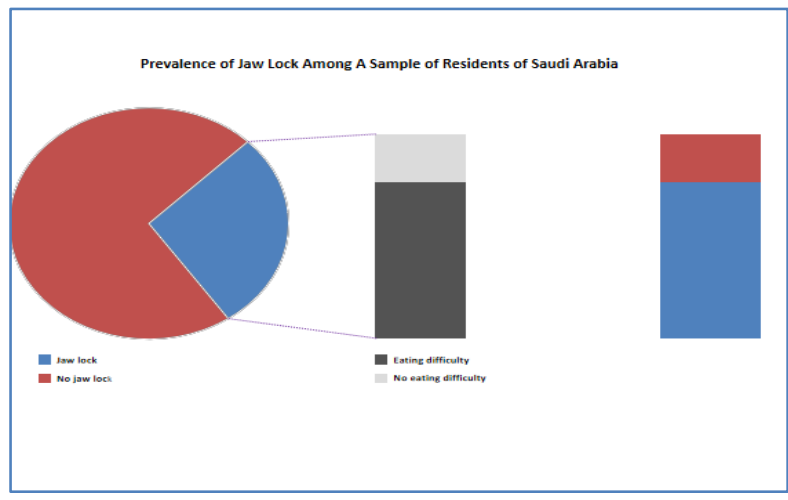


A total of $118(52.2 \%)$ respondents reported suffering orofacial pain during the 30 days that preceded the assessment. Among those, 89 (75.4\%) were females and $29(24.6 \%)$ were males.

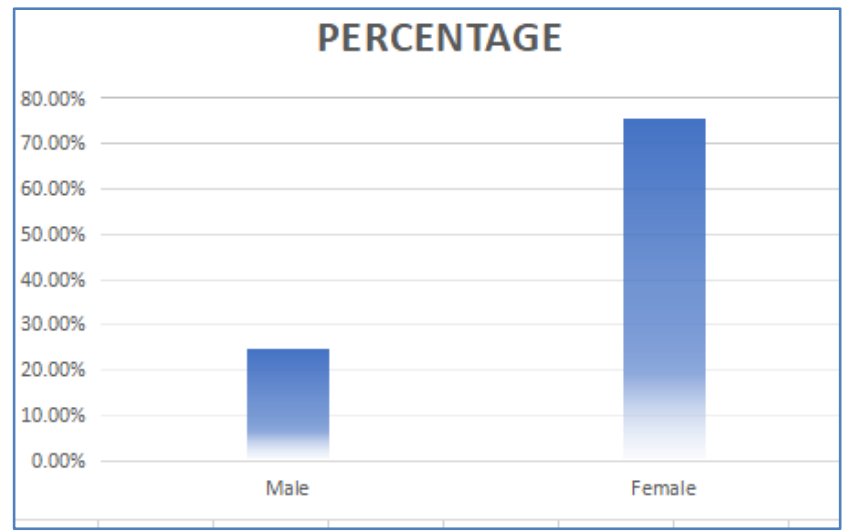

Among those who suffered orofacial pain, 21 (17.8\%) described it as recurring, 34 (28.8\%) described it as sharp, 19/118 continuous, 34/118 sharp, 44 intermittent, 21 recurring. Pain was suffered for an average of $11.2 \pm 5$ months before the present assessment. Patients self-rated their pain at $4.9 \pm 2.6$ in the 10-point visual analogue pain scale. 67 patients described it as recurring in nature and 8 reported constant pain. Axis I related disorders were diagnosed in 195 patients, whereas Axis II related disorders were diagnosed in 36 of patients.

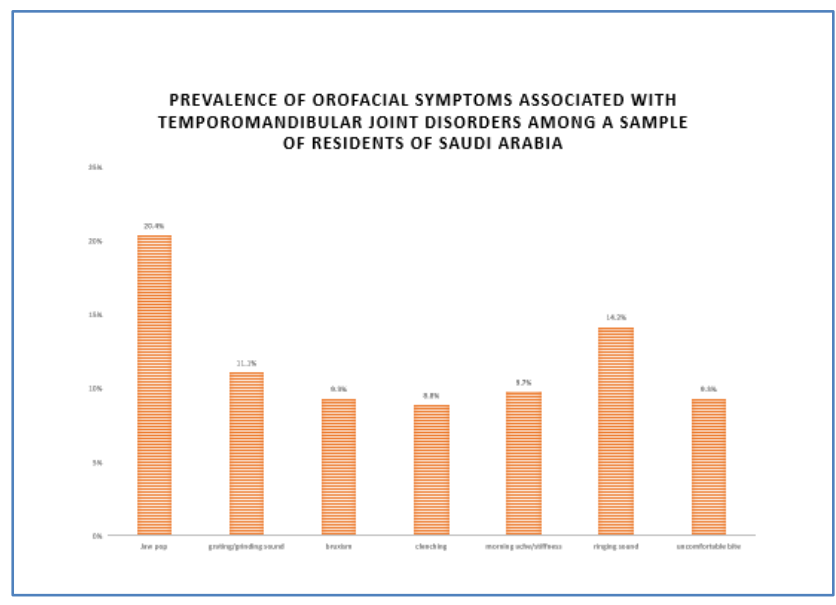

\section{DISCUSSION}

Orofacial pain affects the patients' quality of life. It reduces the quality of their lives by affecting the physical, psychological, and social and function aspects [7]. The patients with orofacial pain are suffering from chronic pain, energy loss and restriction of movement of head and neck [2]. They also suffer from psychological problems like anxiety and depression [2]. All of these difficulties forbid them from practicing their daily social activities in a proper order; as it might be the reason down their frequent absence from the social activities and their work as well due to chronic pain [2].

The signs and symptoms of TMD represent another problem. Is the existence of these signs-or even one of them-sufficient to diagnose TMD? In an attempt to answer this, the Research Diagnostic Criteria for Temporomandibular Disorders (RDC/TMD) questionnaire was developed. This has standardized diagnostic criteria when provided a precise methodology for both examination and diagnosis.

The 1986 Nuprin Pain Report noted that most Americans experience an average of three or four varied kinds of pain annually [7]. Crook and his co-workers reported that $16 \%$ of the general population suffered pain within a 2 -week period. James and colleagues in 1991 reported that greater than $81 \%$ of the population reported an evidentiary jaw pain experience over the course of their lifespan.

Lipton and his co-workers in 1993 noted that $22 \%$ of Americans reported orofacial pain within a 6month time period. Although the orofacial pain most commonly experienced by patients and encountered by oral health care providers is toothache, orofacial pain seldom seems to be an isolated complaint. Turp and 
colleagues noted that more than $81 \%$ of patients reporting to an orofacial pain center had pain sources beyond the trigeminal system. Common comorbid cases include fibromyalgia, chronic fatigue syndrome, headache, panic disorder, gastroesophageal reflux disorder, irritable bowel syndrome, multiple chemical sensitivities, and post-traumatic stress disorder. Often, symptoms for comorbid conditions may distinguish the patient with orofacial pain from patients seeking routine dental care or those with emergent or acute dental pain.

Orofacial pain disorders comprise major and high-priced components of health care in the United States. All together, they have a high prevalence rate and an immense range in pain intensity with commensurate and often significant impact on the quality of life. Ongoing basic and clinical research concentrated on acute and chronic orofacial pain conditions, is necessary to interpret the unique features of this pain system and to develop and evaluate better ways to treat patients with orofacial pain. Additional research is necessary to establish comprehensive classification schemes for all patients with orofacial pain. The present study sample should, therefore, represent different socio-economic patient backgrounds.

One clinical examination protocol for TMD that has gained international acceptance is The Research Diagnostic Criteria for Temporomandibular Disorders (RDC/ TMD), which has been widely used globally to estimate prevalences of TMD. During later years, some limitations with this protocol were identified, and a revised clinical examination protocol has recently been presented, titled The Diagnostic Criteria for Temporomandibular Disorders (DC/ TMD). Some of the items that were questioned in the RDC/TMD were the procedures for diagnosing myofascial pain as well as disc reduction with displacement, and the feasibility and practical application of selected palpation sites.

\section{CONCLUSION}

A multi-disciplinary approach of pain management with the pharmacological and nonpharmacological therapies need to be involved in the orofacial pain management associated with the Head, Face and Neck. If the orofacial pain is not accurately examined, diagnosed, treated and managed can lead to high rate of morbidity, negative socio economic impacts, high health care costs. Proper management of the orofacial pain disorders is done by carefully following the following steps of prevention, diagnosis, treatment, and rehabilitation. It is essential for the clinician and the patient work to unveil all pain sources to improve prognosis and ensure appropriate therapy. Additional research is necessary to establish comprehensive classification schemes for all patients with orofacial pain. The DC/TMD clinical examination protocol appears to be valid for identifying the most common pain-related TMD diagnoses with a reported diagnostic sensitivity of 0.86 and specificity 0.98 .

\section{LIMITATIONS}

This study involves only the population residing in Saudi Arabia, so we cannot generalize our findings to all the population of the Middle East Regions

\section{ETHICAL CONSIDERATIONS Compliance with ethical standards}

Ethical approval: This article contains a survey with human participants performed by all the participants of this research.

Conflict of interest: All the authors do not have any commercial associations that might pose or create a conflict of interest with information presented in this communication. No intramural or extramural funding supported any aspect of this work.

\section{REFERENCES}

1. Baad-Hansen, L., Benoliel, R. (2017). Neuropathic orofacial pain: Facts and fiction. Cephalalgia, 37(7):670-9.

2. Kumar, S., Badiyani, B.K., Kumar, A., Dixit, G., Sharma, P., Agrawal, S.(2016). Orofacial pain and quality of life in early adolescents in India. Int $\mathrm{J}$ Adolesc Med Health, 30(2).

3. Shaefer, J.R., Khawaja, S.N., Bavia, P.F. (2018). Sex, Gender, and Orofacial Pain. Dent Clin North Am, 62(4):665-82.

4. Renton, T. (2017). Chronic orofacial pain. Oral Dis, 23(5):566-71.

5. Racich, M.J. (2018). Occlusion, temporomandibular disorders, and orofacial pain: An evidence-based overview and update with recommendations. J Prosthet Dent, 120(5):678-85.

6. Schiffman, E., Ohrbach, R., Truelove, E., Look, J., Anderson, G., Goulet, J.P. (2014). Diagnostic Criteria for Temporomandibular Disorders (DC/TMD) for Clinical and Research Applications: recommendations of the International RDC/TMD Consortium Network* and Orofacial Pain Special Interest Groupdagger. J Oral Facial Pain Headache, 28(1):6-27.

7. Barros, Vde, M., Seraidarian, P.I., Cortes, M.I., de Paula, L.V.(2009). The impact of orofacial pain on the quality of life of patients with temporomandibular disorder. J Orofac Pain, 23(1):28-37. 